\title{
SURVEY ON LUNG NODULE CLASSIFICATIONS
}

\author{
Ravivarman. $\mathbf{R}^{1}$, Sasirekha. $\mathbf{N}^{2}$ \\ ${ }^{I}$ Student, Communication Systems, Sona College of Technology, TamilNadu, India \\ ${ }^{2}$ Associate Professor, Communication Systems, Sona College of Technology, TamilNadu, India
}

\begin{abstract}
This paper proposes a survey on the classification techniques of lung nodules. We have the different classifications about the nodules in the lungs. It contains the different methods of classification, segmentation and detection techniques. Malignant cell presented in the lungs named, nodules are classified for the treatment processes. Thresholding and Robust segmentation techniques are used in the segmentation process and the feature set is used for classification. Low Dose CT(Computed Tomography) images are applied. This survey has the information about the efficient techniques which are all used for the nodule classification. In these days lung cancer is the dangerous dead disease in the world, So we need to have the knowledge of that cancer. In starting stages the micro nodules are then formed into a cancer cell. Among the cancer affected population about $20 \%$ of the people are dead due to lung cancer. If nodules are found in a starting stage, we can be extend the lifetime of the patient. The main process of this paper involves with the nodule classification and segmentation process of the lung nodules. Here we taken the different procedures involved with nodule detections. CT is the most appropriate imaging technique to obtain anatomical information about lung nodules and the surrounding structures. Here we taken the Low Dose CT(LDCT) images for operations. This paper has the various approaches of the nodule classification. In this survey different techniques are presented which are used for detection and classification of the nodules in the lungs. By differentiating the nodules from the anatomical parts of the lungs, the nodules are identified.
\end{abstract}

Keywords: PLSA, Robust Segmentation and Partitioning.

\section{SEGMENTATION}

Segmentation is the process which is important for the process of Lung Nodule classification and detection. Segmentation is used in partitioning the given input image int0 multiple segments. Here Fan Zhang utilises the thresholding segmentation[1].

There are three segmentations done in the chest LDCT image for lung nodule detection[8]. Here the histogram is computed for the segmentation process, then the smoothing filter is applied to the contour of the segmented image to recover the nodules which are present along with the lung wall. This segmentation consists of three techniques, they are Thorax segmentation, Lung Region Segmentation and Morphological Dilation [8].

Various segmentation techniques are utilized in nodule classification out of which thresholding technique is mostly preferred, as it has no complexity of work and easy for the segmentation over the low dose CT scanned images.

\subsection{Robust Segmentation}

Graph cut based approach [9] and Level set approach [10], which are the two methods which involves robust segmentation algorithms. The objective of these methods is to find the edge of the segmented image. The level set approach function deals with the energy descriptions. In the Graph cut method labeling the volume is according to its gray levels. Then crate a weighted undirected graph with vertices based on volume voxels. This segmentation uses the 3D lung images for 3D segmentation graph. Each vortex in the graph gives the voxel in the lung volume. After thet create the graph for six neighborhoods to compute this segmentation. The energy functions used for formulating and globally minimized to graph cuts [9].

\section{FEATURE EXTRACTION}

For dimensionality reduction we go for the Feature extraction. Feature extraction deals with the image intensity,texture, and gradient. These are used for the various nodule detection and classification in lungs. MR8(Maximum Response)+LBP(local Binary Patterns), Sift descriptor and MHOG (Multiorientation Histogram of Oriented Gradients) are used for the feature extraction process[1]. Used for the feature extraction process four class SVM(Support Vector Machine) is introduced. Generally 128-dimensional feature set is used for the computational process.

\section{CLASSIFICATION}

The low dose CT images are used for the lung nodules detection. There are four types of lung nodules named vascularized, well-circumscribed, juxta-pleural and pleuraltail for lung nodule detection and Fan Zhang gives a context analysis method to classification. This has three main processes (i)An Adaptive patch based division for multilevel partition and (ii)Feature set designing and (iii)Contextual latent semantic analysis based classifier. In the partitioning process a super pixel resolution is introduced to divide the image into sub images. Here they approach the clustering method for partitioning[1]. 
To classify the lung nodules SVM classifier and PLSA (Probabilistic Latent Semantic Analysis) are proposed for the nodule patches and context patches in classification process[1],[4] and SVM classifier is utilized for the probabilistic estimation of the lung nodule [4].

Yan Song approaches the novel classification method for lung nodules. Here also the four types of nodules are classified with the graph construction process. In the optimized graph model global and region based terms are involved and labeling is introduced. Parenchyma, vessel, pleura and pleural-tail are the anatomical parts of the lungs. The foreground is for nodule and background is for the parenchyma and vessel and pleural wall.

Unary term, Pairwise term, Global term and Region-based are the terms used for the global-based voxel labeling. The characterization involves with the SIFT descriptors.This method have the size of 128 dimensional feature set. It used to classify the four types of nodule locations[2].

Another proposal from Farag A, gives the modeling for lung nodule detection. The semiautomatic method of cropping is used and each nodule differentiated into four types of lung nodules. This mehod approches AAM (Active Appearance Modeling) for automatically detecting the lung nodules. Template matching is used for the matching between the AAM model to the original input from the CT scanned image. The Template matching is helped for detecting the lung nodule detection and the intensities of the template and region are computed.This technique gives the model to detecting the nodule from the input of the original image[3].

The problem in the classification process is to classify the overlapped nodule in the anatomical parts of lungs. Here Fan Zhang[4] introduced the improved classification method for overlapping nodes. Here it deals with the Clique Percolation Method (CPM) in the classification of lung nodules. The SVM classifiers are involved and K-means are introduced. Cluster is labeled to the type which has the highest frequency according to the SVM classifier. Most of the the overlapped nodules are between the wellcircumscribed and vascularized[4].For the better nodule classification $\mathrm{K}$ mean is used in SVM classifier. SVM is helped for computing each nodule among the different nodules. Here also the four type of nodules are presented in the lungs and gives classification over the overlapping nodule with the anatomical parts.

The alternate technique for nodule classification is introduced by the Anam Tariq, it examines over the lung tissue to differentiate the nodule from the tissues. In this proposal the Neuro Fuzzy Classifier is introduced. The median filter is introduced to remove noises in the given input during the segmentation process. To removing the back ground we can extract the nodules from the image. By applying Morphological operators we can get the post processed images. Feature extraction process involves to reduce dimensions and used for large number of pixel inputs. Low doseCT scanned images are getting from the public datasets[5].
The another novel method for detecting the lung nodule is to introducing the KNN classifier, and 3D visualization. Here the four process are involved, first step deals with the noise removal by introducing median filter, second process has the feature extraction and classification for detecting lung nodules, next step has active contour model for extracting the contours form the nodule images and the fourth step involves the $3 \mathrm{D}$ visualization for getting good visible results. After the segmentation process median filter the binarization is applied by the adaptive fuzzy thresholding method. In the diagnose process the $3 \mathrm{D}$ visualization is useful for better operations [6] .

S.Ashwin approaches the neural network in the lung nodule detection process, which has sigmoid transfer function and two layered forward pattern. Here the Adaptive Equalization Histogram is applied before the segmentation process. In the equalization process, the thresholding method is applied for the segmentation process to extract the multiple segments from the given input image[7].

\section{DATASET}

Here we use the input images from the Earlier Lung Cancer Action Program (ELCAP)[1]. This dataset has the CT scanned over the lung images. It has the 50 set of low dose CT(LDCT) scanned lung images. These datasets are accessed by the any user through the internet.

\section{CONCLUSION}

Here the survey papers on lung nodule classification are discussed. The detection and classification process which is to be adopted for lung nodule classification are discussed based on the available survey. This paper gives the broader description about the types of lung nodules present in our body and the detection procedures in order to save the human life in early stages.

\section{REFERENCES}

[1]. Fan Zhang ; Yang Song ; Weidong Cai ; Min-Zhao Lee ; Yun Zhou; Heng Huang ; Shimin Shan ; Fulham, M.J. ; Feng, D.D.," Lung Nodule Classification With Multilevel Patch BasedContext Analysis", IEEE transaction on Biomedical Engineering,volume:61, Publication Year: 2014 , Page(s): 1155 - 1166

[2]. Y. Song, W. Cai, Y. Wang, and D. Feng, "Location classification of lung nodules with optimized graph construction," in Proc. Int. Symp. Biomed. Imag., 2012, pp. 1439-1442.

[3]. Farag, A. ; Graham, J. ; Abdelmunim, H. ; Elshazly, S. ; Ei-Mogy, M. ; Ei-Mogy, S. ; Falk, R.; Farag,A.A.,'Small size", Biomedical Engineering Conference (CIBEC), 2012 Cairo International, Page(s): $44-47$.

[4]. F. Zhang,W. Cai, Y. Song, M.-Z. Lee, S. Shan, and D. Feng, "Overlapping node discovery for improving classification of lung nodules," in Proc. Eur. Mol. Biol. Conf., 2013, pp. 5461-5464. 
[5]. Tariq,A. ; Akram,M.U. ; Javed,M.Y.,'Lung nodule detection in CT images using neuro fuzzyclassifier" IEEE Fourth International Workshop on Computational Intelligence in Medical Imaging (CIMI), 2013 ,Page(s): 49 53.

[6]. Soltaninejad,S. ; Keshani,M. ; Tajeripour,F. ,'Lung nodule detection by KNN classifier and active contourmodelling and 3D visualization", 16th CSI International Symposium on Artificial Intelligence and Signal Processing (AISP), 2012 ,Page(s): 440 - 445.

[7]. Ashwin,S. ; Kumar,S.A. ; Ramesh,J. ; Gunavathi,K.,'Efficient and reliable lung nodule detection using a neuralnetwork based computer aided diagnosis system", International Conference on Emerging Trends in Electrical Engineering and Energy Management (ICETEEEM), 2012, Page(s): 135 - 142.

[8]. Farag,A. ; Graham,J. ; Farag,A.,"Robust segmentation of lung tissue in chest CT scanning", 17th IEEE International Conference on Image Processing (ICIP), 2010 , Page(s): 2249 - 2252.

[9]. Asem Ali and Aly A. Farag, "Automatic Lung Segmentation of Volumetric Low-Dose CT Scans Using Graph Cuts," $4^{\text {th }}$ Int. Sym. on Visual Comp (ISVC-08), Las Vegas, Dec. 1-3,

2008, pp. 258-267.

[10]. Aly A. Farag and Hassan Abdelmunim (2004), "Adaptive segmentation of multi-modal 3D data using robust level set techniques," in Proc. Int. Conference on Medical Image Computing and Computer-Assisted Intervention (MICCAI' 04), Saint Malo, France, Sept. 2629, 2004, pp. 143-150.

\section{BIOGRAPHIES}

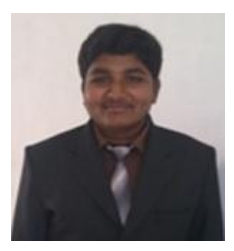

Ravivarman. $\mathrm{R}$ was born in Tamilnadu,India in 1992.Currently he is a student of sona college of technology in communication systems.

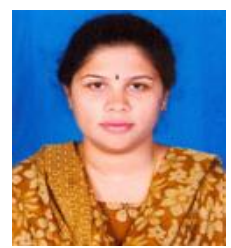

Sasirekha.N is cureently a professor of sona college of technology in communication systems. 